XII.

Aus der chirurgischen Cunversitätsklinik Heidelberg (Geh. Rat Narath.)

\title{
Die Bedeutung der Adduktoren für das Hüftgelenk mit Berücksichtigung der übrigen auf dieses Gelenk wirkenden Muskeln.
}

Von

Dr. Roith.

Mit 1 Abbildung im Text, 10 Abbildungen und 42 Kurven auf Tafel XX-XXVII.

Sowohl die rationelle Behandlung der meisten Frakturen als auch besonders die Transplantationsversuche ron Sehnen und Muskeln zur Korrektion der verschiedenen Lähmungen bedingen ein steigendes Interesse des praktischen Chirurgen an der mechanischen Wertigkeit einzelner Muskelindividuen.

Die grundlegenden diesbezüglichen Arbeiten Duchenne's über die Physiologie der Bewegungen geben uns keine genügende Beantwortung der Fragen, welche uns die beiden oben genannten immer grösser werdenden Disziplinen der Chirurgie stellen. Die tatsächliche, rasche Entwicklung dieser Disziplinen beweist ihre bereits vollzogene zum Teil noch sich rollziehende Abtrennung und Sonderung als besondere Spezialität. In höherem Grade noch als die Behandlung der Frakturen sind es die funktionelle Diagnose und die orthopädischen Operationen bei Lähmungen, welche ein tieferes Verständnis der Statik und Mechanik des Gelenk- und ibres Muskelapparates bedingen.

Trotzdem ist uns ein Verfahren zu einer mechanisch-mathematischen Analyse einzelner Muskelgruppen und Individuen von theoretischer Seite gegeben worden in der Arbeit: Über die Statik und Mechanik des Schultergürtels unter normalen und pathologischen Verhältnissen von S. Mollier.

In dieser Arbeit hat der Autor durch die Konstruktion eines bewegungsmechanischen Modells die Wirkungen der einzelnen Muskeleinheiten die Synergeten, die Antagonisten und die kombinierte Tätigkeit sämtlicher den Schultergürtel bewegenden Muskeln exakt reranschaulicht und auf Grund der so gewonnenen Resultate Messungsmethoden angegeben, welche uns mit einfachen mechanischen Hilfsmitteln eine exakte Diagnose aller Lähmungen an den genannten Bezirken gestatten und zwar genauer als es uns die elektrische Untersuchung ermöglicht. 
Bei dem grossen klinischen Interesse, das das Hüftgelenk bietet, erschien es mir angebracht, die Muskeln desselben nach ähnlichen Methoden und Prinzipien zu untersuchen besonders aber eine Muskelgruppe, die man unter dem Namen der Adduktoren zusammenfasst, deren Wirkungsweise auf das Hüftgelenk aber durchaus nicht mit einem Wort charakterisiert werden kann.

Schon die einfache Betrachtung einer unteren Extremität sowohl am anatomischen Präparat als auch in vivo lässt es auffallend erscheinen, dass für die Adduktion allein eine so beträchtliche Muskelmasse vorbanden sein soll, dass sie der der Extensoren und Flexoren des Unterschenkels gleichkommt, Dabei besorgt aber beim Stehen die Adduktion ron jeder Abduktionsstellung bis zur Vertikalen an sich schon dje Schwerkraft, ohne dass eine Unterstïtzung durch Muskelkraft notwendig wäre. Eine Adduktion von der Vertikalen medialwärts ist eine relativ seltene Bewegung. Auch die Regulation der Schwingungsebene des schreitenden Beins erfordert keine solche enorme Muskelmasse sondern wird ebenfalls grösstenteils durch die Schwerkraft besorgt.

Die Grösse der Adduktorengruppe beweist aber, dass diesen Muskeln eine grosse mechanische Aufgabe obliegt und diese können wir nur darin suchen, dass die Adduktoren entweder auch anderen ständigen Bewegungen der unteren Extremität dienen oder dass die Adduktion über die Vertikale hinaus viel häufiger ist als wir bisher annahmen.

Eine Erfahrung aus dem praktischen Leben führt uns zur Annahme des erstgenannten Moments. Es ist dies der Adduktorenschmerz nach intensiven Beugebewegungen z. B. Bergsteigen, Fussballspiel und ähnlichen.

In den meisten mir zugänglichen Lehr- und Handbüchern der Anatomie werden die Adduktoren als ausschliesslich dieser Funktion dienende Muskeln bezeichnet.

Dagegen analysiert Duchenne die Funktion dieser Muskeln auf Grund faradischer Reizung wie folgt:

1. Durch den Pectineus wird der Oberschenkel gegen das Becken gebeugt und etwas nach einwärts gebracht, gleichzeitig führt er um seine Längsachse eine Rollbewegung von innen nach aussen aus.

2. Durch den Adductor brevis wird der Oberschenkel gleichfalls gebeugt und nach innen gebracht, da aber die dabei stattfindende Adduktionsbewegung stärker ausgesprochen ist als seine Bewegung nach vorne, so wird er durch den Oherschenkel der anderen Seite in dieser Bewegung aufgehalten. Der Muskel bewirkt ausserdem eine Rollbewegung von innen nach aussen.

3. Durch den Adductor magnus wird der Oberschenkel gerade nach innen gebracht, ausserdem bewirkt sein oberer Abschnitt eine Drehung des Schenkels von innen nach aussen, während sein unterer Abschnitt ihm eine Rollbewegung von aussen nach innen erteilt, um ihn in seine normale Stellung zurückzuführen, wenn er sich vorher in Rotation nach aussen befand.

Was den Adductor longus angeht, so ist er vom Pectineus und Adductor brevis bedeckt und der direkten Faradisation unzugänglich.

Unter dem Einfluss der Faradisation des Gracilis wird die Unterextremität in kräftige Anziehung versetzt. 
Auch Cruveilhier hält den Pectineus, Adductor brevis und auch magnus für Muskeln, die eine bedeutende Beugekomponente aufs Hüftgelenk besitzen.

Ein weiterer Anhaltspunkt für die Richtigkeit dieser Annahme ist in den Innervationsverhältnissen der Adduktoren zu finden. Der Pectineus bezieht nämlich wie auch bisweilen der Adductor longus seine Nerven aus dem Nervus cruralis, der auch den Rectus femoris und sartorius versorgt. Da die letzteren Muskeln Beuger im Hüftgelenk sind, liegt nach Analogie der Innervationsverhältnisse die Vermutung nahe, dass den erst genannten Muskeln auch diese Funktion zukommt.

Aus den pathologischen Zuständen der Lähmungen des N. obturatorius ersehen wir nichts, was zur gewünschten Klärung beitragen könnte. Im allgemeinen machen diese Lähmungen geringe Störungen (von Strümpell). Sie werden selten beobachtet. Ich habe während meiner mehrjährigen gynäkologischen Tätigkeit zwei Durchschneidungen des N. obturatorius bei ausgedehnten Freund'schen Operationen erlebt. Die beiden intelligenten Patientinnen wurden sich nach ihrer Genesung ihrer Lähmungen gar nicht bewusst.

Wir ersehen aus dem Vorstehenden, dass nach dem gegenwärtigen Stand unserer anatomischen und klinischen Kenntnisse und Erfahrungen eine eingehende Untersuchung über die Wirkungsweise der sämtlichen Muskeln des Hüftgelenks besonders der Adduktoren berechtigt ist. Bevor ich mich aber der Methode der mathematischen Analyse ihrer Funktion zuwende, möchte ich darauf verweisen, dass die gegebenen Grössen keine absoluten, sondern relative Werte darstellen, da die Faktoren grossen Schwankungen unterworfen sind, welche vom Individuum, dem Geschlecht, der Rasse und häufigen pathologischen Zuständen abhängen. Ich erinnere an die Variabilität des Beckens an dem die Muskeln entspringen je nach Geschlecht, Rasse, bei Rachitis, Osteomalacie, ferner bei Erkrankungeu der Wirbelsäule, ferner an die Variabilität des Oberschenkelhalses unter denselben Verhältnissen, endlich der Beckenneigung. Besonders letztere hat auf die folgenden Untersuchungen einen wesentlichen Einfluss.

Ich habe meine Untersuchungen an drei verschiedenen Objekten ausgeführt und dabei gefunden, dass die Schwankungen wohl beträchtlich aber keine prinzipiellen sind.

Eben wegen der grossen Variabilität einerseits, ferner um andererseits durch die Schilderung komplizierter Untersuchungsmethoden den Kliniker nicht unnötig zu langweilen, habe ich die Methode Mollier's etwas vereinfacht, trotzdem ich ihren Prinzipien treu geblieben bin. Dazu hielt ich mich um so mehr berechtigt als die Funktionsbestimmung der Muskeln eines Kugelgelenks ein viel einfacherer Vorgang ist, als Ähnliches am Schultergürtel, ferner da Variabilitäten vor allem pathologische Knochenformen am Hüftgelenk ausserordentlich viel häufiger sind als am Schultergürtel.

Ich wende mich der Schilderung des Verfahrens zu, durch welches ich die Wirkungskomponenten der Adduktoren auf die Frontal- und Sagittalachse des Hüftgelenkes zu ermitteln versuchte. Da es mir nicht um den 
mechanischen Effekt der Muskelkontraktion, sondern um die Ermittelung von Komponenten zu tun war, konstruierte ich einen Apparat, der es mir ermöglichte, bei jeder beliebigen Stellung des Beins die Spannungsverhältnisse der zu untersuchenden Muskeln abzulesen. Ich verfuhr dabei wie folgt:

An einer normalen unteren Extremität mit erhaltener Beckenhälfte und Lendenwirbelsäule wurden sämtliche Adduktoren, ferner der Semitendinosus, Semimembranosus und Biceps präpariert, die übrige Muskulatur entfernt. Ursprung und Ansatz der genannten Muskeln am Knochen wurden besonders genau präpariert. Jeder Muskel wurde nun je nach seiner Grösse durch einen oder mehrere starke Fäden ersetzt, welche genau dem Verlauf der Muskelfasern entsprechen. Der Faden war am distalen Ansatzpunkt befestigt und

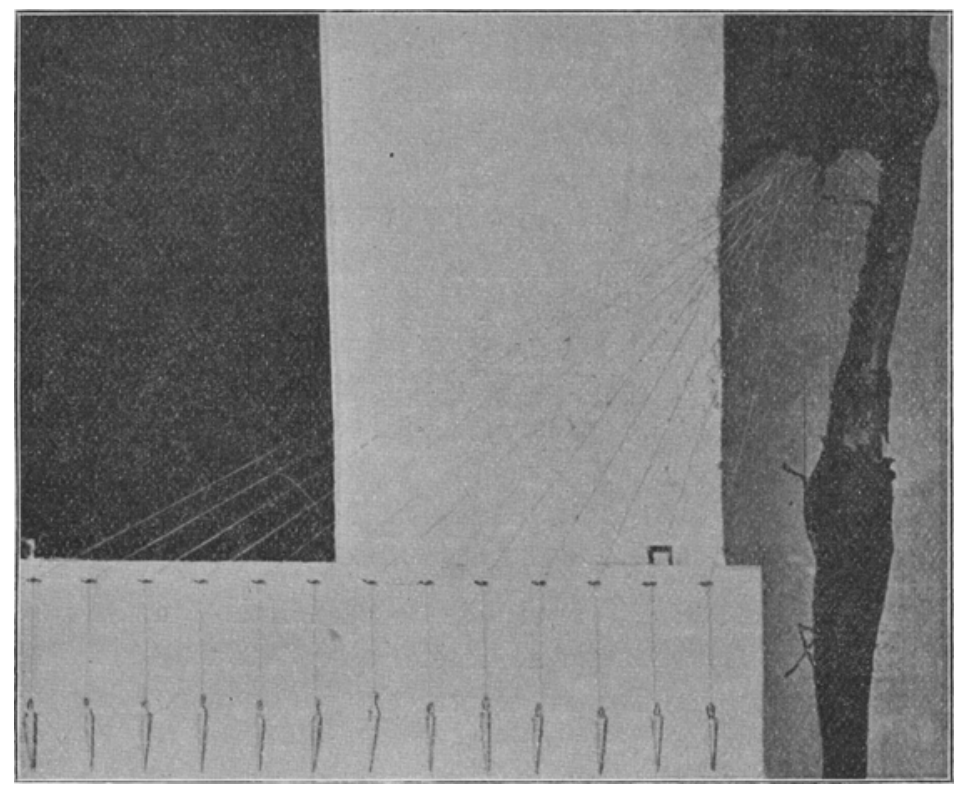

Fig. 2.

zwar beim Semitendinosus, Semimembranosus, Gracilis und Biceps ${ }^{1}$ ) an der Sehne, bei den übrigen Muskeln direkt am Knochen. Entsprechend dem proximalen Ansatzpunkt des Muskels waren eine oder mehrere kleine Metallösen in den Knochen eingeschraubt, durch welche die entsprechenden Fäden liefen. Um die Verkürzung und Verlängerung der Fäden bei den verschiedenen Bewegungen zu registrieren, bediente ich mich eines glatten senkrecht aufgehängten Brettes von $40: 50 \mathrm{~cm}$, an dessen oberem Rand in Abständen von $2 \mathrm{~cm}$ kleine Metallösen horizontal eingeschraubt waren. Durch diesen liefen die Fäden, welche in der Mitte des Brettes endigten. Um sie in Spannung zu halten, wurden an die Enden Bleistücke gehängt. Sie wurden so eingestellt, dass sie bei Ruhelage der Extremität, mit der sie verbunden waren, sich in einer Horizontallinie auf der Mitte des Brettes befanden. Wurden nun bei fixiertem Brett und bei einer der dem aufrechten Stehen 1) Caput longum. 
entsprechenden Lage und bei Fixation des Beckens an der zu untersuchenden Extremität Bewegungen im Hüftgelenk ausgeführt, so wurden die Fäden dadurch ge- oder entspannt, wodurch eine Verschiebung der Gewichte in der Vertikalen auf dem Brett bewirkt wurde. Da die Verschiebungslinien in Zentimeter geteilt waren, so kann man auf dem Brett für jede Stellung der Extremität die Grösse der Verlängerung oder Verkürzung der zu untersuchenden Muskeln direkt mit einem Blicke in Zentimeter ablesen. Ferner sieht man, je nachdem die Verschiebung der Gewichte in gleicher oder entgegengesetzter Richtung statthat, welche Muskeln für die jeweilige Bewegung Synergeten, welche Antagonisten sind. Findet bei irgend einer Bewegung eine Verschiebung nach abwärts statt, so sind die Muskeln, welche durch diese Fäden ersetzt werden, Synergeten der jeweiligen Bewegung im umgekehrten Fall Antagonisten. (Fig. 1 und 2.)

Der Apparat beruht auf demselben Prinzip, wie der, den Mollier zur Erforschung der Statik und Mechanik des Schultergürtels angegeben hat. (Festschr. f. C. v. Kupffer, Jena 1899).

Da ich mich aber erst im Laufe der Arbeit dazu entschloss, die Untersuchungen auch auf die übrigen Muskeln des Hüftgelenks auszudehnen und für die einzelnen Muskeln und Muskelabschnitte Bewegungskurven darzustellen, verfuhr ich nun etwas anders. Zunächst ersetzte ich die übrigen in Betracht kommenden Muskeln entsprechend der Resultante - oder Resultanten (bei gefiederten Muskeln 7. B. Glutaeus medius) - durch Schnüre in der eben geschilderten Weise.

Als registrierender Apparat diente mir ein glattes Brett. Dasselbe wurde an einem Schreibeapparat horizontal vorbeigeführt, welcher an einer senkrecht aufgehängten, durch zwei Ösen verlaufenden, unten beschwerten Schnur lief. Diese Schnur wurde an ihrem oberen Ende mit der den jeweiligen $\mathbf{z u}$ untersuchenden Muskel ersetzenden verbunden. Um gleichmässige Kurven zu erhalten, war es aber notwendig, dass sich die Bewegung der Extremität, welche sich durch die verschiedenen Schnüre auf den Schreibapparat übertrug, in allen Bewegungsphasen mit der gleichen Geschwindigkeit abspielte, wie die Bewegung des am Schreibeapparat vorübergleitenden Brettes. Dies erreichte ich dadurch, dass ich letzteres durch eine über Rollen laufende Schnur mit einem bestimmten Punkt der zu untersuchenden Extremität verband. An die entgegengesetzte Seite des Brettes wurde eine ebenfalls über Rollen laufende Schnur mit einem daranhängenden Gewicht befestigt. Bewegte man nun die Extremität, so wurde hierdurch nicht nur der Schreibeapparat, sondern auch die zu beschreibende Tafel in gleichem Masse bewegt.

Es wurden nun von jeder Schnur zwei Kurven gezeichnet: Eine für die Bewegung um die horizontale Frontalachse von einer Flexion von $90^{\circ}$ bis zu einer Extension von $30^{\circ}$. Eine zweite für die Bewegung um die horizontale Sagittalachse von $60^{\circ}$ Abduktion bis ca. $30^{\circ}$ Adduktion. Der Punkt, der der Ruhelage der ruhig hängenden Extremität entsprach, wurde auf jeder Kurve markiert. Durch diesen Punkt wurde eine zur gezogenen Abszisse parallele Linie gelegt. Man kann so ohne weiteres aus dem Verlauf und der Höhe 
der Kurve die Art der Bewegung des in Frage kommenden Muskels von der Ruhelage aus ersehen.

Die erhaltenen Kurven wurden auf $1 / 3$ verkleinert. Es zeigt also die Höhe der vertikalen Abszisse ein Drittel der tatsächlichen Verlängerung oder Verkürzung des in Frage kommenden Muskels an.

\section{Resultate der Messungen.}

Im vorstehenden Abschnitt will ich den individuellen von den zahlreichen genannten Momenten abhängigen Massergebnissen Rechnung tragend nicht Zahlen, sondern Bewegungskurven miteinander vergleichen. Ich will gleich vorausschicken, dass die Kurven der auch aufs Kniegelenk wirkenden Muskeln wie Biceps, Semitendinosus, Rectus, Gracilis, Sartorius bei im Knie gestreckter Extremität aufgenommen sind und dass sich diese Kurven bei einer Lageveränderung im Kniegelenk natürlich verändern und zwar so, dass bei Beugung die Kurven der flektierenden (im Knie) Muskeln niedriger, der extendierenden höher werden. Die Kurven sind von links nach rechts zu lesen. Bei den Beugekurven entspricht das linke Ende einer Beugung am Hüftgelenk von ca. $90^{\circ}$, das rechte einer Streckung von ca. $30^{\circ}$, bei den Kurven der Bewegung um die sagittale Horizontalachse das linke Ende einer Abduktion von ca. $60^{\circ}$. das rechte einer Adduktion von ca. $30^{\circ}$. Der Schnittpunkt der Kurve und der sie schneidenden Abszisse entspricht der Ruhelage $=$ Lage der Extremität am hängenden und annähernd aufrechtstehenden Körper.

Bei den Kurven bedeutet der Verlauf derselben oberhalb der durch den Ruhepunkt laufenden Abszisse eine Verlängerung der zu untersuchenden Resultante, der Verlauf unterhalb der Abszisse eine Verkürzung derselben.

Die Bewegungs-Kurven um die horizontale Frontalachse, welche von links unterhalb der Abszisse nach rechts oberhalb der Abszisse verlaufen, sind als Beugungs-Kurven, diejenigen, welche von links oben nach rechts unten verlaufen, als Streckungs-Kurven zu bezeichnen.

Diejenigen Kurven der Bewegung um die horizontale Sagittalachse, welche von links unten nach rechts oben verlaufen, sind Abduktoren, die von links oben nach rechts unten verlaufen, Adduktoren entstammend.

Die Kurven sind um so steiler, je weiter der Becken-Ansatzpunkt der zu untersuchenden Muskelresultante von der durch die horizontale frontale resp. sagittale Bewegungsachse gelegten Vertikalebene entfernt ist, je günstiger also die mechanischen Momente für die Muskelwirkung sind.

Je steiler die Kurve, desto günstiger und wirksamer ist der Zug an dem zu bewegenden Objekt im Sinne der Zugrichtung. Es gilt dies natürlich sowohl für die ganze Kurve, wie auch für die einzelnen Abschnitte derselben.

Gleichsinnig verlaufende Bewegungskurven (derselben Achse) sind Synergeten, sich schneidende Antagonisten bildenden Muskeln entsprungen. 
Der rotatorischen Komponente der Muskeln des Hüftgelenks wurde keine Beachtung geschenkt, da dieselbe hinreichend in allen Lehrbüchern der Anatomie gewürdigt wird.

Untersucht wurden alle Muskeln des Hüftgelenks. Jene Kurven aber, die von benachbarten gleichartig wirkenden Muskeln abstammen und im Verlauf geringe unwesentliche Differenzen aufwiesen, sind zum Teil weggelassen, z. B. die des Semitendinosus conf. Kurve des Semimembranosus, des Pyriformis conf. hintere Partie des Glut. med. Die beiden Obturatorii haben sehr geringe Höhe der Beugungs- und Adduktionskurven, sie sind hauptsächlich Rotatoren, ebenso die Gemelli. Diese Kurven bleiben aus ökonomischen Rücksichten weg.

Ich wende mich nun der Schilderung der einzelnen Bewegungskurven, die bei Bewegung der Extremität um die horizontale Sagittalachse aufgenommen wurden, zu.

Gleichsinnig verlaufend, also Synergeten entstammend, sind die Kurven sämtlicher Adduktoren des Gracilis, des Pectineus, des Semimembranosus, des Semitendinosus (nicht aufgeführt, da der des Semimembranosus völlig gleich), des Biceps und des Quadratus femoris. Es sind also diese sämtlichen Muskeln im Besitz einer mehr minder grossen Adduktionskomponente.

Antagonisten zur obengenannten Gruppe sind die Glutäen (Glutaeus minimus fehlt, Kurve ähnlich der des medius), ferner der Rectus, der Sartorius und der Tensor fasciae latae. Alle diese Muskeln besitzen also eine abduktorische Komponente.

Eine Sonderstellung nimmt der Ileo-psoas ein (conf. Fig. 3). Seine Resultante verläuft ungefähr durch die horizontale Sagittalachse des Hüftgelenks. Es fehlt ihm also eine Wirkung auf diese Achse in der Ruhelage. Befindet sich dagegen die Extremität in Ab-oder Adduktion, so wird er dadurch passiv gedehnt, und er wird bei Verkürzung bestrebt sein, das Bein in die Ruhelage zurückzubringen. Doch ist, wie aus dem flachen Verlauf der Kurve hervorgeht, seine diesbezügliche Komponente sehr gering und seine Zugrichtung für eine derartige Bewegung ungünstig. Er kommt praktisch nicht in Betracht. Beim breiten weiblichen Becken besitzt der Psoas eine geringe abduktorische Komponente von der Ruhelage aus.

Abduktoren. (Conf. Fig. 3.)

Die steilste Kurve dieser Muskelgruppe besitzt der M. tensor fasc. latae. Er zeigt bei der Abduktion des Beines von der Ruhelage eine grössere Exkursionsweite und günstigere mechanische Verhältnisse, als bei dem Ausgleich bestehender Adduktion wie aus der Kurve hervorgeht.

Ihm folgt der vordere Abschnitt des M. glut. medius. Doch ersehen wir aus der Kurve, dass die Exkursionsweite und die mechanischen Verhältnisse für die Abduktion vom Ruhepunkt und für den Ausgleich einer bestehenden Adduktion ungefähr die gleichen sind. Die Ähnlichkeit der Kurven dieser beiden Muskeln erklärt sich auch aus der Ähnlichkeit ihres anatomischen Verlaufes. 
Der Sartorius zeigt ebenfalls eine steile aber doch eine niedrigere Kurve als seine beiden Vorgänger. Vor allem ist zu bemerken, dass er fast nur der Abduktion aus der Ruhelage dient, während seine Bedentung für den Ausgleich einer bestehenden Adduktion sehr gering anzuschlagen ist.

Die mittlere und hintere Partie des G]utaeus medius sind fast gleichwertig, die Bedeutung der letzteren scheint etwas geringer als die der ersteren. Auch diese beiden Muskelabschnitte dienen in erster Linie der Abduktion rom Ruhepunkt.

Es folgt der Rectus mit einer ansehnlichen Abduktionskurve rom Ruhepunkt aus und einer minimalen Wirkung zum Ausgleich bestehender Adduktion.

Die Abduktionskomponente des Glutaeus maximus ist nicht gross. Seine Wirkung zum Ausgleich bestehender Adduktion steht der reinen Abduktionswirkung wenig nach. Wenn man aber seine enorme Masse in Betracht zieht, so kann man wohl annehmen, dass es sich um recht bedeutende Effekte handelt. Ich habe zur Darstellung seiner Wirkungsweise deshalb nur eine dem Verlauf seiner mittleren Fasern entsprechende Resultante gewählt, weil sein Faserverlauf ein fast völlig paralleler ist, also in allen Teilen gleichartige Wirkung vorausgesetzt werden darf.

\section{Adduktoren. (Conf. Fig. 4.)}

Der Besprechung der hierher gehörigen Kurven muss ich vorausschicken, dass es bei der Analyse der mechanischen Funktionen der Adduktoren im engeren Sinne nicht möglich ist, den in der deskriptiven und topographischen Anatomie : als einzelnes Muskelindividuum figurierenden Adductor magnus (auch maximus) als solches zu charakterisieren. Schon bei der Betrachtung dieses Muskels am anatomischen Präparat geht aus dem ungleichmässigen Faserverlauf dieses Muskels mit Deutlichkeit hervor, dass seine mechanische Bedeutung. in den einzelnen Abschnitten eine recht verschiedene sein muss. Die z. T. sich kreuzende Richtung seiner Fasern findet nach Analogie des Pectoralis major ihre Erklärung darin, dass hier wie dort eine grosse fächerförmige Muskelmasse, die beim Adductor magnus peripher, fast der ganzen Medialseite des Femur entlang ihren Ansatz findet, zentral nur einen kleinen Haftpunkt entlang des unteren Sitzbeinastes hat und es dann zu einer Einrollung der vorher flach ausgebreiteten Muskelfasern kommt. Dass die Einrollung tatsächlich hesteht, lässt sich am anatomischen Präparat leicht demonstrieren, wo man sie durch Präparation entfalten kann. Aus dem mehr minder divergenten Verlauf der Fasern der einzelnen Muskelabschnitte geht hervor, dass ihre Wirkung in mancher Richtung eine antagonistische sein muss, dies gilt besonders von den Bewegungen um die frontale Horizontalachse.

Vom mechanischen Standpunkte teilt man den Muskel meines Erachtens am besten in eine vordere ventrale und hintere dorsale Fasergruppe, und diese einzelnen Gruppen wieder in kurze und lange oder mehr schräg oder senkrecht verlaufende Fasern. Bei dieser Einteilung schliesst sich die vordere Partie des Adductor max. mehr der vorderen Gruppe der Adduktoren im 
engeren Sinn, die hintere Partie dieses Muskels yor allem die langfaserige, fast senkrecht verlaufende zwangslos der medialen Gruppe der an der Hinterseite des Oberschenkels gelegenen Muskeln i. e. semitendinosus und semimembranosus an. Da nun der Faserverlauf eines Muskels uns die Richtung seiner Wirkung angibt, liegt schon nach der einfachen Besichtigung des anatomischen Präparates der Schluss nahe, dass die Funktion des hinteren Abschnittes der Adductor max. der des semimembranosus und semitendinosus gleicht, während die vorderen Abschnitte eine der Funktion der vorderen Adduktorengruppe ähnliche besitzen. Die Richtigkeit dieser Spekulation wird durch das Folgende bestätigt. Details über den Verlauf der Adduktoren finden sich in den meisten guten neueren Atlanten und Lehrbüchern der Anatomie, so dass ich an dieser Stelle von einer Rekapitulation dieser Dinge absehen zu dürfen glaube.

Die Resultanten der einzelnen Adduktoren wählte ich folgendermassen. Gracilis, Pectineus und Quadratus femoris wurde durch je einen Strang ersetzt. Der Adductor maximus durch vier, entsprechend der oben bereits erwähnten Einteilung. Adductor brevis und longus wurden durch je zwei den Resultanten ihrer oberen und unteren Abschnitte entsprechende Stränge ersetzt.

Die grösste Adduktionskomponente zeigen nach dem Verlauf der Kurven die unteren Fasern des Adductor brevis und die oberen und unteren Fasern des Adductor longus. Die Kurven hauptsächlich der beiden ersteren, die ja auch am Präparat fast genau denselben Faserverlauf zeigen, sind fast identisch. Es besteht nur ein kleiner Unterschied zwischen dem Teil (oberer) der Kurve, welche den Ausgleich der Abduktion und dem (unterer) der die Adduktion von der Ruhelage aus darstellt, aus dem steileren Verlauf des letzteren ergibt sich, dass hiebei die mechanischen Verhältnisse für die Muskelwirkung, soweit sie von der Richtung der angreifenden Kraft abhängen, hier etwas günstiger sind als dort. Die unteren langen Fasern des Adductor longus zeigen bereits den der Adduktion von der Ruhelage aus entsprechenden Teil der Kurve zugunsten des die Ausgleichungsmöglichkeit der Abduktion darstellenden etwas verkürzt.

Ähnlich verhalten sich die oberen kurzen Fasern des Adductor brevis. Der flachere Verlauf der Kurve weist darauf hin, dass die Kraft dieses Muskels unter ungünstigeren Verhältnissen d.h. unter einem spitzeren Winkel an der zu bewegenden Extremität angreift.

Die Kurve der kurzen vorderen Fasern des Adductor maximus und des Gracilis zeigen fast dasselbe Verhalten.

Bereits wesentlich flacher sind die Kurven der vorderen langen Fasern des Adductor maximus und des Pectineus. Dies weist auch darauf hin, dass sich die mechanisch ungünstigen Verhältnisse der Richtung der Krafteinwirkung noch gesteigert haben. Diese Muskeln weisen unter der vorderen Gruppe die ungünstigsten Verbältnisse auf, wenn man vom Quadratus femoris absieht, dessen Wirkung bei der geringen Muskelmasse wohl nicht wesentlich anzuschlagen ist. Nur beim Pectineus ist der dem Ausgleich der Abduktion entsprechende Teil der Kurve gleich dem der Adduktion von der Ruhelage aus entsprechenden. Bei den übrigen übertrifft ersterer den letzteren beträchtlich. 
Die Adduktionskurve der am und in der Nähe des Tuber ischii entspringenden Muskeln ist beträchtlich flacher und um das zwei- bis dreifache niedriger als die der vorn entspringenden Muskeln.

Die hinteren kurzen Fasern des Adductor maximus weisen ungefähr dieselbe Kurve auf wie die vorderen langen. Noch flacher ist die der hinteren langen. Nach dem Verhalten der Kurve scheinen diese Abschnitte hauptsächlich die Adduktion des abduzierten Beines zu besorgen und an der Adduktion vom Ruhepunkt aus kaum und nur unter ungünstigen mechanischen Bedingungen (Angriff der Muskelkraft unter kleinen Winkeln) beteiligt zn sein. Es fällt die grosse Ähnlichkeit der Kurve der unteren hinteren Fasern des Adductor maximus mit den Kurven des Semimembranosus (Semitendinosus) und Biceps auf, welche eine noch flachere Kurve aufweisen.

Ich komme nun zur Betrachtung der Kurven, welche bei Bewegung des Hüftgelenks um die frontale Horizontalachse aufgenommen wurden. Nach der Gleichartigkeit erweisen sich als Synergeten im Sinne einer Beugung im Hüftgelenk der Ileopsoas, der Rectus femoris, Tensor fasciae latae, Sartorius, Glutaeus medius vordere Fasern, (Pyriformis) ${ }^{1}$ ), Pectineus, Adductor longus lange Fasern, Adductor brevis kurze Fasern; Synergeten im Sinne einer Streckung im Hüftgelenk sind: der Biceps, Semimembranosus (Semitendinosus), die sämtlichen Fasern des Adductor max., besonders die dem Semi-tendinosus und membranosus zunächst gelegenen, der Quadratus femoris und die mittleren und hinteren Fasern des Glutaeus medius und des Glut. maximus.

Endlich finden sich Kurven, welche eine Flexions- und Extensionskomponente besitzen, so die des Gracilis, des Adductor longus (kurze Fasern), des Adductor brevis (lange Fasern), bisweilen die des Quadratus femoris und die vorderen Fasern des Adductor maximus, besonders die kurzen. Das Vorkommen einer Flexionskomponente beim Quadratus fem. und den vorderen Fasern des Adductor maximus liegt im Bereich individueller Schwankungen. Jedenfalls sind diese Komponenten so gering, dass ihnen eine praktische Bedeutung wohl fehlen wird.

\section{Die Kurven der Beuger. (Fig. 5.)}

Die steilsten Beugekurven weisen der Tensor fasciae latae und der Sartorius auf. Wie aus den Kurven hervorgeht, dienen die beiden Muskeln vor allem der Beugung der Extremität von der Ruhelage aus. Es geschieht dies unter den günstigsten mechanischen Bedingungen, wie aus der Steilheit des diesbezüglichen Kurvenabschnittes deutlich hervorgeht. Die Komponente zum Ausgleich bestehender Streckung ist gering, die mechanischen Bedingungen zur Vollbringung dieses Momentes sind ungünstig, d. h. die Muskelwirkung vollzieht sich unter einem sehr spitzen Winkel, wie aus der Kurve ersichtlich ist. -

1) Kurven nicht dargestelit. 
Der Rectus femoris und der Ileopsoas zeigen ähnliche, nur etwas flachere Kurven. Im übrigen gilt für diese das über die beiden rorigen Muskeln gesagte. Doch ist zu bedenken, dass der Rectus, besonders aber der lleopsoas eine ganz gewaltige Muskelmasse darstellt und dass sie wohl aus diesem Grunde die bedeutendsten Beugemuskeln sind.

Der Pectineus zeigt eine ähnliche, nur etwas flachere Kurve als der Psoas; eine noch flachere zeigen die vorderen Fasern des Glut. medius. Die langen Fasern des M. adductor longus und die kurzen des Adductor brevis zeigen eine geringe Beteiligung an der Beugung des Beines von der Ruhelage aus. Der grössere und steilere T'eil der Beugungskurve entspricht dem Teil der Kurve, der die Beteiligung dieser Muskeln am Ausgleich bestehender Streckung darstellt. Die mechanischen Verhältnisse für die Beugung sind nur zu Beginn dieser Bewegung günstig, je mehr sich die Beugung dem rechten Winkel nähert, desto spitzer wird der Winkel der Angriffsrichtung dieser Muskeln, so dass von einer Beugung von ca. $30^{\circ}$ ab ihre Beugekomponenten bedeutungslos sind. Die Kurve des Adductor brevis (kurze Fasern) ist flacher und niedriger als die der langen Fasern des Add. longus. Es überwiegt also die mechanische Bedeutung des letzteren.

\section{Die Kurven der Strecker. (Fig. 6.)}

Semimembranosus (Semitendinosus), Biceps und die hinteren oberen und unteren Fasern des Adductor maximus zeigen nahezu identische Kurven. Es sind dies die steilsten Kurven der Strecker. Der grössere Teil der Kurven entfällt auf den Teil, der die Streckung des maximal gebeugten Beines bis zum Ruhepunkt darstellt, der kleinere auf den Teil, der der Streckung vom Ruhepunkt aus entspricht. Doch ist der erstere Teil der Kurve flacher, der letztere wenigstens zum Teil steiler, so dass sich hieraus die Tatsache ergibt, dass die Streckung vom Nullpunkt aus unter mechanisch günstigeren Bedingungen erfolgt, als die Streckung des gebeugten, vor allem des extrem gebeugten Beines.

Auch der Glutaeus maximus zeigt eine ähnliche, aber wesentlich niedrigere Kurve. Hier ist der Unterschied der Steilheit der Kurven noch auffälliger, so dass man wohl annehmen kann, dass er sich an der Streckung des extrem gebeugten Beines infolge seines in dieser Stellung zu der zu bewegenden Extremität fast völlg parallelen Verlaufes kaum beteiligt. Die mechanischen Verhältnisse zur Entfaltung seiner Wirkungen werden mit der zunehmenden Streckung immer günstiger. Zur Beurteilung seiner grossen mechanischen Bedeutung bedarf es des Hinweises auf seine enorme Muskelmasse.

Den mittleren und hinteren Fasern des Glutaeus med. kommt eine geringe Streckkomponente zu.

Die langen und kurzen vorderen Faserndes Add. maximus, ferner der Quadratus femoris zeigen eine von den vorhergehenden etwas abweichende Kurvenform. Bei sämtlichen zeigt der der Streckung des extrem gebeugten Beines entsprechende Kurventeil einen gleichmässigen, ziemlich steilen Ver- 
lauf, so dass die grosse Bedeutung dieser Muskeln für die Streckung des extrem gebeugten Beines klar erhellt. Die Kurve wird, je mehr sie sich dem Ruhepunkt nähert, flacher, woraus sich die rasche Abnahme der Bedeutung dieser Muskeln für die weitere Streckung ergibt. Der Quadratus femoris zeigt überhaupt keine Komponente für die Streckung vom Ruhepunkt aus. Im Gegenteil, es erhellt aus der Kurve, dass er bei weiterer Streckung über seinen toten Punkt hinaus, allerdings mit einem minimalen Ausschlag, einer weiteren Streckung entgegenwirkt, dass er also eine Streck- und Beugekomponente in sich vereinigt. An einem anderen Objekt fand ich dieses Verhalten auch an den vorderen Fasern des Add. max., vor allem an den kurzen. Die Inkonstanz dieses Befundes hängt von der individuellen Variabilität, vielleicht auch von der etwas verschiedenen Wahl der Resultante ab. Es kommt jedoch dieser Variation eine prinzipielle Bedeutung nicht zu. So viel können wir sicher sagen, dass diese Muskeln mit der zunehmenden Streckung an Wirksamkeit verlieren und gegen Ende der Streckung für dieselbe bedeutungslos werden. Der individuelle schwankende Umschlag der Wirkung in eine antagonistische ist nur von theoretischer Bedeutung, wenn man die Anordnung der Muskeln ums Hüftgelenk vom Standpunkt der mechanischen Zweckmässigkeit aus betrachtet.

Es finden sich jedoch mehrere Muskeln und Muskelabschnitte an der Hüfte, welche derartige antagonistische Komponenten besitzen. Es ist dies vor allem der Gracilis. Es ist dies aus seiner Kurve und aus einer einfachen Überlegung seiner durch seine Topographie zum Hüftgelenk bedingten mechanischen Verhältnisse leicht zu ersehen. Die Kurve sagt uns, dass der Gracilis auf das in Ruhelage befindliche Bein (bei Streckung im Knie) eine geringe Beugung unter mechanisch wenig günstigen Bedingungen ausübt, daraus geht hervor, dass der tote Punkt dieses Muskels sehr nahe dem Ruhepunkt liegt, dass er also das Bestreben hat, extreme Bewegungen um die horizontale Frontalachse durch seine Kontraktion auszugleichen. Er besitzt auch eine ganz ansehnliche Streck- und Beugekomponente für das extrem gebeugte resp. gestreckte Bein. Die mechanischen Bedingungen für die Entfaltung seiner Wirkung sind um so günstiger, je weiter die Extremität von der Ruhelage entfernt ist.

Vergegenwärtigen wir uns seinen Verlauf und seine Topographie zum Gelenk in den verschiedenen Stellungen, so können wir auf diese Weise die Richtigkeit der Kurve ziemlich genau kontrollieren.

Das dem aufrechten Stand entsprechend aufgehängte Muskelpräparat einer unteren Extremität mit erhaltenem Beckenring zeigt uns, dass der Gracilis GB die Achse der unteren Extremität AB unter einem sehr spitzen Winkel schneidet und sein Ansatzpunkt G etwas unter dem Drehpunkt des Gelenks A und nicht weit von der durch die horizontale Frontalachse gelegten Vertikalebene des Hüftgelenks gelegen ist. Aus dieser Anordnung erhellt, dass der Muskel nach einer kurzen Exkursion im Sinne der Beugung von der Ruhelage aus auf seinem toten Punkt AGC angelangt ist und dass durch eine weitere Beugung der Extremität der Muskel in eine passive Spannung versetzt wird, die ent- 
sprechend der Exkursion zunimmt und dass dabei der Winkel, den er mit der Extremität bildet, immer grösser wird (Fig. 7). Gleichzeitig kann man auch erkennen, welche grosse Bedeutung unter Umständen die Beckenneigung für die Funktion eines Muskels haben kann (Fig. 8). Ist die Beckenneigung steiler, so rückt der Insertionspunkt des Gracilis in der Ruhelage der Extremitätenachse immer näher, trifft dieselbe und fällt eventuell hinter sie. Ist sie geringer rückt er immer mehr von ihr ab. Im letzteren Fall wird die beugende Komponente vom Ruhepunkt aus auf Kosten der strecken. den grösser. Im ersteren kleiner, unter Umständen Null, oder es rückt sogar die streckende Komponente bis hinter den Nullpunkt.

Diese Erörterung gilt natürlich im Prinzip für alle Muskeln. Ich habe sie hier eingeflochten, da sich diese Beobachtung am Präparat am Gracilis besonders leicht ohne irgendwelche Hilfsmittel machen lässt.

Die langen unteren Fasern des Adductor brevis und die kurzen oberen des Adductor longus zeigen ein ähnliches Verhalten. (Fig. 9.) Doch ist die Streckkomponente für das gebeugte Bein hier bedeutend kleiner, besonders beim Adductor longus. Die Hauptwirkung dieser Muskeln auf die frontale Horizontalachse scheint die Beugung, besonders des gestreckten Beines, zu sein. Sie geht auch unter den günstigsten mechanischen Verhältnissen vor sich. Bezüglich der Beugungskurvenkomponente gilt hier auch besonders das, was eben beim Gracilis üher die Beziehung derselben zur Beckenneigung gesagt wurde.

Die Bewegungskurven für die anderen Vertikalebenen ausser der frontalen und sagittalen, lassen sich für die einzelnen Muskeln ohne weiteres berechnen, daher hielt ich es für überflüssig, dieselben mit dem Apparat zu zeichnen und den schon gegebenen noch hinzuzufügen.

Es hätten sich natürlich sämtliche Kurven durch Berechnung konstruieren lassen, nachdem die Länge jedes Muskels in der Ruhelage, der Abstand seiner peripheren Insertion an der Fixtremität rom Drehpunkt im Hüftgelenk und der seiner zentralen Insertion ron demselben Gelenk leicht durch Messung bestimmt und daraus die Kurve ohne weiteres für die Verkürzung und Verlängerung des in Frage kommenden Muskels bei einer beliebigen Stellung und Bewegung ermittelt werden kann. Doch hielt ich den von mir eingeschlagenen Weg für den praktikableren, um so mehr als es uns weniger auf ganz genaue Werte als auf Typen, aus den bereits erwähnten Gründen, ankommt.

Die ursprünglich geplante Konstruktion eines bewegungsmechanischen Modells nach den Mollierschen Prinzipien, durch welches man die Bewegungen, welche durch die einzelnen Muskeln und Muskelabschnitte hervorgerufen werden, naturgetreu nachahmen kann, stiess auf erhebliche technische Schwierigkeiten, auch bekäme man bei einem Gelenk mit so grosser Exkursionsbreite viel ungenauere Werte, als dies bei den Gelenken des Schultergürtels der Fall ist, die im Verhältnis zum Hüftgelenk viel geringere Exkursionsbreiten und bestimmtere Bewegungsbahnen besitzen. Ebenso erforderte 
die Beschreibung der Resultate, die man mit einem bewegungsmechanischen Modell erzielt, eine viel umfänglichere Bearbeitung, ohne dass dabei in unserem Fall die praktische Bedeutung derselben eine wesentliche Förderung erfahren hätte.

Einen einzigen Vorteil gewährt die Beobachtung am bewegungsmechanischen Morlell, nämlich den, dass man für jedes Stadium der Bewegung aus dem Stand der die Muskeln ersetzenden Marken die Antagonisten und Synergeten der jeweiligen Bewegung und ihre jeweilige Inanspruchnahme ohne weiteres ablesen kann. Ich gebe nur von den uns hier besonders interessierenden Adduktoren einige diesbezügliche Tafeln. Ich muss hervorheben, dass hier die Verkürzung und Verlängerung in einem anderen Massstab und nicht wie in den Kurven auf $1 / 3$ reduziert, wiedergegeben ist. Die Tafeln sind mit dem erst geschilderten Apparat aufgenommen.

Die auf der horizontalen Abszisse der beiliegenden Tafel stehenden Zahlen bedeuten die einzelnen Muskelpartien und zwar die Zahlen 1-4 den Adductor magnus. 1 entspricht den vorderen Partien des Muskels, welche steil nach abwärts verlaufen, 2 den Fasern, welche vorne entspringen und zum oberen Abschnitt der Linea aspera verlaufen. 3 und 4 entsprechen den hinteren Partien, die in nächster Nähe des Tuber oss. ischii entspringen und zwar 3 den steil nach abwärts und 4 den mehr horizontal verlaufenden Faserbündeln, 5 entspricht dem Gracilis, 6 dem Semimembranosus, 7 dem Semitendinosus, 8 dem Biceps; bei diesen Muskeln blieb das periphere Sehnenstück erhalten und diente zum Haftpunkt des Fadens. 9 entspricht der untersten Partie des Adductor longus, 12 der obersten des Adductor brevis, 10 und 11 den dazwischen liegenden Partien der genannten zum Teil sich überdeckenden Muskeln, 13 den mittleren Fasern des M. pectineus.

Betrachten wir nun an der Hand der von meinem registrierenden Apparat gegebenen Bilder die Wirkung der Adduktoren, ferner des M. semitendinosus, -membranosus und biceps auf die um die sagittale Horizontalachse bewegte Extremität.

Ich lasse der Einfachheit halber die Bilder von der extremsten Abduktion zur extremsten Adduktion folgen, da bei dieser Reihenfolge manches einfacher ist, als wenn wir die Spannungsverhältnisse der Muskeln bei von der Ruhelage aus ausgeführter Ad- und Abduktion betrachten.

Bei einer Abduktion von ca. $85^{\circ}$ (Fig. 10) sind alle genannten Muskeln passiv gespannt, d. h. sie sind Antagonisten zur Abduktion und besorgen die Adduktion des so abduzierten Beines, welche im praktischen Leben, z. B. beim seitlichen Schritt, zugleich die Seitwärtsbewegung des Körpers zu leisten hat, zusammen mit grosser Kraft, am intensivsten der Adductor magnus und von seinen einzelnen Abschnitten der vordere untere am stärksten. Er hat in dieser Stellung eine Dehnung von $10 \mathrm{~cm}$ erfahren, während dieselbe bei den anderen Abschnitten $6^{1 / 2}-7^{1 / 2} \mathrm{~cm}$ beträgt.

Die intensivste Dehnung weist der Gracilis mit 11 auf.

Auch Semimembranosus, -tendinosus und Biceps haben eine Dehnung von $6 \mathrm{~cm}$ erfahren. Etwas mehr sind der Adductor longus und der untere Abschnitt des Adductor brevis beteiligt, während der obere Abschnitt des 
letztgenannten Muskels eine Dehnung von nur $4 \mathrm{~cm}$, der Pectineus von nur $2 \mathrm{~cm}$ erfahren hat.

Ist nun die Abduktion bis auf ca. $45^{\circ}$ vermindert (Fig. 11), so ist die Dehnung des Adductor magnus schon um $21 / 2-4 \mathrm{~cm}$ reduziert, ebenso die des Gracilis. Die Muskelgruppe auf der Rückseite des Oberschenkels ist um $4 \mathrm{~cm}$ entspannt, während die Spannung der oberen Adduktoren (longus, brevis, pectineus) kaum abgenommen hat. - Mit der Rückkehr in die Normallage ist die passive Spannung ausgeglichen.

Bei einer Adduktion von ca. $20^{\circ}$ sind in geringem Masse die vordere obere und untere Partie des Adductor magnus durch aktive Kontraktion $=$ Verkürzung der Fäden (Ausschlag unter die Linie der Ruhestellung) beteiligt, während sich die hinteren Partien dieses Muskels an dieser Bewegung nicht mehr beteiligen. (Fig. 12.)

Auch Gracilis und Semimembranosus tragen durch geringe Kontraktion zu dieser Adduktion bei. Die Adduktionskomponenten von Semitendinosus und Biceps sind erschöpft, während die obere Gruppe der Adduktoren durch Kontraktion den grössten Anteil an dieser Bewegung hat.

Bei maximaler Adduktion von ca. $35^{\circ}$ (Fig. 13) sind die hinteren Partien des Adductor magnus wieder zu Antagonisten geworden, sie sind im Zustand geringer passiver Dehnung, ebenso die Muskeln an der Rückseite des Femur, während der Adductor longus, brevis und pectineus durch ausgiebige Kontraktion an dieser Stellung beteiligt sind. In geringerem Masse der Gracilis und die vordere obere Partie des Adductor magnus, am wenigsten die vordere untere Partie des genannten Muskels.

Daraus ergibt sich, dass der Adductor magnus hauptsächlich zur Adduktion des abduzierten Beines dient, während die hinteren Partien desselben, welche am Tuber ischii in der Nähe des Semitendinosus, membranosus und Biceps entspringen, ebenso wiediese, Antagonisten einer Adduktion über die Normallage sind; nur die vorderen Partien des Muskels besitzen noch eine geringe adduktorische Komponente. Die Adduktion von der Normallage aus besorgen vor allem der Adductor longus, brevis und pectineus.

Bei Betrachtung der einzelnen Bewegungsphasen um die frontale Horizontalachse finden wir folgendes:

Bei extremer Beugung von ca. $80^{\circ}$ (Fig. 14) des (im Knie gestreckten) Beins sind der Adductor magnus, der Gracilis und die an der Rückseite des Oberschenkels gelegene Muskelgruppe in extremster passiver Spannung, d. h. sie sind Antagonisten zur Beugung und besorgen die Streckung des so gebeugten Beins, welche im praktischen Leben z. B. beim Vorwärtsschreiten zugleich die Vorwärtsbewegung des Körpers zu leisten hat. Die passive Spannung dieser Muskeln ist hierbei ebenso gross, wie bei maximaler Abduktion. Am wenigsten ist der Gracilis und die rordere obere Partie des M. adductor magnus dabei beteiligt, mit einer Dehnung von $6 \mathrm{~cm}$, während die übrigen Partien des letztgenannten Muskels, die in der Nähe des Tuber 
oss. ischii entspringenden und die vordere steil nach abwärts verlaufende, ferner der Semitendinosus usw. eine Dehnung von 9-10 cm erfahren. Die oberen Adduktoren sind bei der Beugung entspannt, d. h. sie unterstiutzen die Beugung durch ihre Kontraktion und behindern die Streckung, am meisten der M. pectineus und die obere Partie des Adductor brevis (Entspannung um 2,5-3,5 cm), in geringerem Grad der Adductor longus und der untere Abschnitt des Adductor brevis (1-1,5 cm Entspannung).

Bei einer Beugung von ca. $50^{\circ}$ (Fig. 15) ist die Streckungskomponente der erstgenannten Muskeln um fast die Hälfte, die des Gracilis fast völlig aufgebraucht, während die Beugungskomponente der oberen Adduktoren auf $3 \mathrm{~cm}$ Verkürzungsmöglichkeit angewachsen ist, nur die des M. pectineus hat um $1 \mathrm{~cm}$ abgenommen.

Bei einer Beugung von ca. $20^{\circ}$ besitzt auch der Gracilis eine geringe Beugekomponente.

Bei der maximalen Extension (von ca. 25 ${ }^{\circ}$, Fig. 16) endlich besitzen nur mehr noch die hinteren Partien des M. Adductor magnus, dann die Muskelgruppe an der Hinterseite des Oberschenkels eine geringe Streckkomponente, indem sie gegen die Ruhelage eine Verkürzung von $1,5-2 \mathrm{~cm}$ aufweisen. Die vorderen Partien des Adductor magnus, der Gracilis und die oberen Adduktoren sind in dieser Stellung kräftige Antagonisten zu einer weiteren Streckung, am wenigsten die vordere untere Partie des Adductor magnus und der Pectineus $(0,5-1,5 \mathrm{~cm}$ gedehnt), am meisten Gracilis, Adductor longus und brevis $(3-4 \mathrm{~cm}$ gedehnt).

Auch bei den Bewegungen um die horizontale Frontalachse ist der Adductor magnus, vor allem seine hinteren Partien, ein Synerget von Semitendinosus und den beiden anderen Muskeln. Nur seine vorderen Partien besitzen eine geringe flektorische Komponente auf das gestreckte Bein, während die hinteren Partien reine Strecker sind. Eine geringe Flexion, bis zu $15-20^{\circ}$ bewirkt der M. gracilis. Im höchsten Masse sind der Adductor longus, brevis und M. pectineus Beuger. Sie erzielen eine Beugung von $20-30^{\circ}$. Auf das maximal gebeugte Bein wirkt der Adductor magnus stark extendierend.

Auf einen Punkt möchte ich noch hinweisen, dass nämlich bei den uns interessierenden Muskeln, wie überhaupt bei denen der unteren Extremität, bald der zentrale, bald der periphere Insertionspunkt das Punctum fixum ist, worauf wir bei der Würdigung ihrer Funktionen und bei der Prüfung ihrer mechanischen Zweckmässigkeit stets achten müssen.

\section{Zusammenfassung.}

Überblicken wir kurz die einzelnen Muskelgruppen, zusammengestellt nach der Gleichartigkeit ihrer Bewegung und ihre Bedeutung für den Ablauf derselben in den einzelnen Bewegungsphasen.

Ich beginne mit den Beugern. Mustern wir ihre Kurven, so sehen wir, dass ihr Anteil an der Beugung von der Ruhelage aus viel bedeutender 
ist, als an der Beugung des gestreckten Beins bis zur Ruhelage. Nur bei wenigen unbedeutenden Muskeln überwiegt die letztgenannte Komponente. Gegen Ende der maximalen Beugung nimmt die Steilheit der Kurven ab, d. $h$. die beugende Kraft wird geringer, z. T. verwandelt sie sich in eine antagonistische. Diese Anordnung ist ausserordentlich zweckmässig, da die Beugung des Beines von der Ruhelage aus der Schwere entgegen bewerkstelligt werden muss, wogegen die Beugung des gestreckten Beines zur Ruhelage im Sinne der Schwerkraft sich vollzieht. Die Abnahme der beugenden Kraft oder ihr Umschlag in eine antagonistische gegen das Ende der Bewegung ist zweckmässig wegen der Verhinderung einer Hyperflexion, wegen der dadurch bedingten Schonung der Gelenkkapsel und wegen der beim Schritt nun folgenden Streckung. Die beugende Kraft darf gegen Ende der Bewegung abnehmen, da letztere zum Teil schon durch das Beharrungsvermögen weiter geführt wird. Die Beuger dienen vielmehr der Bewegung der Extremität gegen das Becken, als der umgekehrten. Letztere bedarf keines grossen Muskelapparates, da sie sich meist im Sinne der Schwerkraft vollzieht. Übrigens ist das tiefe Vorwärtsbeugen des Körpers, z. B. beim Aufheben eines Gegenstandes eine Bewegung, die sich eben so sehr im Lumbalteil der Wirbelsäule wie im Hüftgelenk abspielt, die ferner noch durch Beugung im Knie kräftig unterstïtzt wird.

Bei den Streckern finden wir ein umgekebrtes Verhalten. Hier ist bei den meisten Muskeln der auf die Streckung des gebeugten Beins entfallende Teil der Kurve der grössere und steilere, wogegen der Abschnitt der Kurve, der der Streckung von der Ruhelage aus entspricht, kürzer und flacher ist. Nur beim Glutaeus maximus sind die beiden Abschnitte annähernd gleich, daraus erhellt, dass der Streckung des gebeugten Beines eine bedeutendere Rolle zufällt als der Streckung ron der Ruhelage aus und dass jene einer grösseren Muskelmasse bedarf als diese. Die Zweckmässigkeit dieser Einrichtung wird klar, wenn wir uns die Mechanik des Ganges grobschematisch vergegenwärtigen. Der erste Teil des Schrittes ist das Vorsetzen des im Hüftgelenk gebeugten Beines. Strecken wir nun das vorgesetzte Bein im Hüftgelenk, so wird dadurch der Körper vorwärts bewegt. Die Vorwärtsbewegung des Körpers beim Gehen wird also vor allem durch die Streckung des gebeugten Beines erzielt. Dabei wird, je nachdem der Körper beim Gehen dauernd vorwärts gebengt wird, eine Streckung über den Ruhepunkt nur im geringem Grade oder gar nicht stattfinden. Daraus erhellt das quantitative und qualitative Überwiegen der Streckkomponente des gebeugten Beines uber die vom Ruhepunkte aus. Erstere hat beim Gang, soweit Bewegungen im Hüftgelenk in Betracht kommen, die Hauptaufgabe. Auch hier finden wir, dass die Zahl der Synergeten im Beginne der Bewegung am grössten ist und gegen das Ende derselben abnehmen resp. in einen z. T. sogar beträchtlichen Antagonismus umschlagen. Allerdings ist dieser letzte Moment bei der Streckung von untergeordneter mechanischer Bedeutung, da die Gelenkkapsel gegen die Überstreckung durch einen gewaltigen Bandapparat (Lig. ileofemorale u. a.) geschützt ist. 
Hervorzuheben ist die grosse Bedentung eines Teiles der Adduktoren für die Streckung (conf. vorne).

Bezüglich der Abduktoren ist zu bemerken, dass sie hauptsächlich der Abduktion von der Ruhelage aus dienen, weniger dem Ausgleich einer bestehenden Adduktion, welcher durch die Schwerkraft allein erzielt werden kann.

Umgekehrt wirken die Adduktoren hauptsächlich zum Ausgleich einer bestehenden Abduktion. Ihre reine Adduktionskomponente (d. h. Adduktion von der Ruhelage betreffend) ist kleiner als die ebengenannte. Es erscheint demnach der Schluss berechtigt, dass die ab- und adduktorischen Komponente hauptsächlich zur Equilibration des Spielbeins und der entsprechenden Körperhälfte dienen um beim Gang die Schwankungen, um die horizontale Sagittalachse $\mathrm{zu}$ vermeiden und ihn so stabiler zu machen.

Die Adduktoren wirken also auf die frontale und Sagittalachse des Hüftgelenkes fast in gleichem Masse. Sie sind so angeordnet, dass sie z. T. die Beugung der Extremität im Hüftgelenk von der Ruhelage aus im Beginn der Bewegung synergetisch mit dem M. ileopsoas u. a. unterstützen. Die synergetische Wirkung nimmt aber proportional der Grösse der Exkursion $\mathrm{ab}$ und verwandelt sich $\mathrm{z}$. T. bei zunehmender Beugung in eine antagonistiscbe. Bei der entgegengesetzten Bewegung des Schrittes, bei dem die vorgesetzte Extremität die ganze Körperlast vorwärts zu bringen hat, also bei der Streckung im Hüftgelenk ist demzufolge ebenfalls im Anfang der Bewegung und zwar proportional der Grösse der Exkursion die Zahl der Synergeten am grössten unter den mechanisch günstigsten Verbältnissen. Mit der zunehmenden Streckung im Hüftgelenk nimmt aber die Zahl der diesbezüglichen synergetischen Komponenten ab. Der Winkel, unter dem sie auf die Extremität wirken. wird kleiner, also die mechanischen Verhältnisse ungünstiger und schliesslich verwandeln sich z. T. die ursprünglichen Synergeten am Ende der Extension in Antagonisten.

Dieselben Verhältnisse finden wir bei der Bewegung um die Sagittalachse (also bei der $\mathbf{A b}$ - und Adduktion), doch wird diese nicht allein von den Adduktoren, sondern auch rom Semitendinosus, semimembranosus und caput longum des Biceps besorgt und bei Bewegungen um die Vertikalachse (Aus- und Einwärtsrotation).

Demnach ist die Gruppierung der Adduktoren um das Hüftgelenk als eine mechanisch ausserordentlieh zweckmässige zu bezeichnen, da z. T. dieselben Muskelgruppen, welche im Anfang einer Bewegung dieselbe unterstützen, am Schluss derselben Bewegung, wenn schon die Trägheit der Masse wirkt, hemmend eingreifen und so das Gelenk vor unnötigen mechanischen Insulten bewahren.

Bei dem praktischen Versuch, die Eigenschaft der Adduktoren als Strecker des Hüftgelenks zu steigern - was nach dem anatomischen Verhalten dieser Muskeln jedoch nur schwer zu erreichen wäre - musste man von der Überlegung ausgehen, dass die Streckkomponente dieser Muskeln zunimmt, je mehr man den Ursprung der Adduktoren nach vorne verlegt und dass hierbei die Adduktionskomponente kleiner wird. Dasselbe erzielt 
man durch die laterale Verschiebung der Adduktorenursprünge. Doch darf man nicht übersehen, dass bei den ebengenannten Manipulationen, mit dem Grösserwerden der Streckkomponente die zwangsweise mit der Streckung verbundene Auswärtsrotation zunimmt.

Ferner ist es zweifellos, dass der dauernde mechanische Reiz, den die Adduktoren auf das $O_{s}$ pubis und Os ischii ausüben, einen gestaltenden Einfluss auf das Becken haben muss und dass es beim Wegfall desselben zu Beckendeformitäten kommen muss.

Eigentlich gehört nun nach der qualitativen Analyse der Muskelfunktionen noch eine quantitative. Eine solche liesse sich auf Grund von Wägungen, Längs- und Querschnittsmessungen der analysierten Muskeln und Muskelabschnitte an einem grossen Material und aus daraus berechneten Mittelwerten approximativ aufstellen. Das war mir leider nicht möglich. Aber trotz dieser und anderer Mängel hoffe ich, dass die Resultate dieser Arbeit gelegentlich bei der Betrachtung der Bewegungen im Hüftgelenk unter den verschiedenen pathologischen Zuständen und bei der Therapie der letzteren Berücksichtigung finden. Vielleicht ist es mir vergönnt, auf diese Kapitel selbst zurückzukommen. 